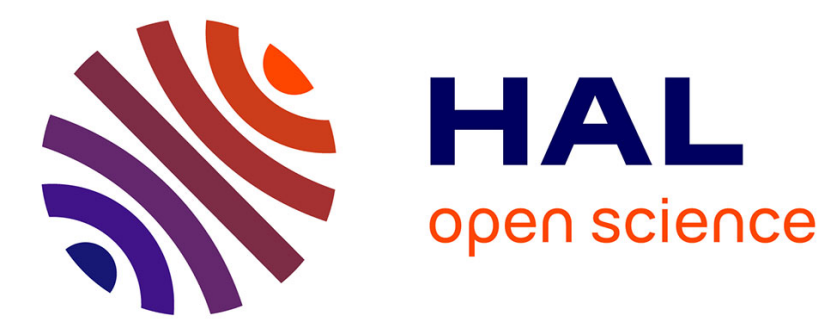

\title{
Self-similarity in urban wireless networks: Hyperfractals
}

Philippe Jacquet, Dalia Popescu

\section{To cite this version:}

Philippe Jacquet, Dalia Popescu. Self-similarity in urban wireless networks: Hyperfractals. Workshop on Spatial Stochastic Models for Wireless Networks (SpaSWiN), May 2017, Paris, France. hal01498987

\section{HAL Id: hal-01498987 \\ https://hal.inria.fr/hal-01498987}

Submitted on 30 Mar 2017

HAL is a multi-disciplinary open access archive for the deposit and dissemination of scientific research documents, whether they are published or not. The documents may come from teaching and research institutions in France or abroad, or from public or private research centers.
L'archive ouverte pluridisciplinaire HAL, est destinée au dépôt et à la diffusion de documents scientifiques de niveau recherche, publiés ou non, émanant des établissements d'enseignement et de recherche français ou étrangers, des laboratoires publics ou privés. 


\title{
Self-similarity in urban wireless networks: Hyperfractals
}

\author{
Philippe Jacquet $^{1}$, Dalia Popescu ${ }^{1,2}$ \\ ${ }^{1}$ Nokia Bell Labs, ${ }^{2}$ INRIA \\ France \\ Email:\{philippe.jacquet,dalia-georgiana.herculea\}@nokia-bell-labs.com
}

\begin{abstract}
We introduce a model of Poisson patterns of fixed and mobile nodes on lines designed for urban wireless networks. The pattern obeys to "Hyperfractal" rules of dimension larger than 2. The hyperfractal pattern is best suitable for capturing the traffic over the streets and highways in a city. We show that the network capacity under ad hoc routing algorithms scales much better than with the classic uniform Poisson shot model. The scaling effect depends on the hyperfractal dimensions. We show this results in two different routing models: nearest neighbor routing with no collision, minimum delay routing model assuming slotted Aloha and signal to interference ratio (SIR) capture condition, power-path loss and Rayleigh fading. The novelty of the model is that, in addition to capturing the irregularity and variability of the node configuration, it exploits self-similarity, a characteristic of urban wireless networks.
\end{abstract}

\section{INTRODUCTION}

Future networks require challenging and diverse communication scenarios with topologies designed to fit specific usecases [1], [2]. The explosion of the Internet of Things (IoT), devices employing ad-hoc communication has brought back in the attention the ad-hoc networks. Traditionally, for the modeling of ad-hoc topologies, uniform Poisson spatial models are employed. These models have been successfully applied to analyze wireless networks that exhibit a high degree of randomness. The characteristics and network metrics have been extensively studied in scientific literature [3] and results about the scaling laws are well-known [4].

Urban infrastructures display a certain degree of regularity that has been modeled by the Manhattan grid [5], used as a lattice with users positioned in its corners. In reality, users have random positions on streets and the density of the users on each street depends on the level of importance of the respective street in the city road map (boulevard, street, alley, etc).

Recently, models of fractal repartition have been introduced [6], [7] and have shown that environment displays selfsimilarity characteristics. For example, a department has rural areas, with low density of population, and urban areas, with high density of population, namely cities and towns. The towns are split in neighborhoods, each neighborhood is organized in blocks separated by streets. Blocks are made of buildings that are themselves split in apartments and so on. This description is very close to a fractal object that is based on self-similarity. Figure 1 represents Indianapolis downtown road map and is a perfect illustration of a map exhibiting self-similarity properties.

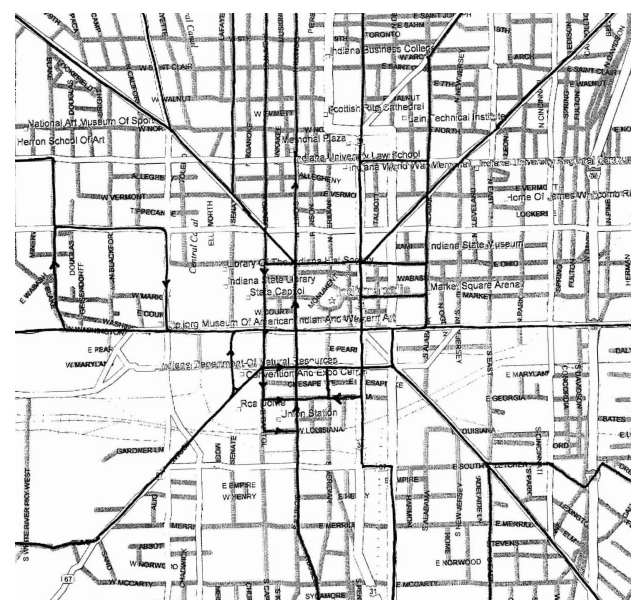

Figure 1: Indianapolis downtown road map

Results that exploit self-similarity are very promising. In [6], the authors showed that a limit of the capacity in a network with a non-collaborative protocol is inversely proportional to the fractal dimension of the spatial repartition of terminals. In their model the nodes have locations defined as a Poisson shot inside a fractal subset, for example a cantor set.

By definition a fractal dimension is smaller than the Euclidean dimension; it can be arbitrary smaller. In this work we propose a new model, which we call "Hyperfractal", for the ad-hoc urban wireless networks. This model captures not only the irregularity and variability of the node configuration but the self-similarity of the topology as well. The hyperfractal model is not a Poisson shot model in a fractal support but rather is a Poisson shot model which has support a measure which has scaling properties. It is a kind of generalization of fractal Poisson shot models, and in some cases, in fact in every case of our urban traffic models, it will have a dimension that is larger than the Euclidean dimension and this dimension can be arbitrarily large.

The radio model comprises urban-specific phenomenons such as the "urban canyon" propagation effect which is the most realistic in urban context.

Using insights from stochastic geometry and fractal geometry, we derive scaling laws of information routing metrics as well of throughput capacity and we prove by numerical analysis and simulations the accuracy of our expressions. 


\section{System Model AND GeOMETRY}

\section{A. Urban Geometry: hierarchical grid street model}

Let us assume that map is the unit square and it is divided into a grid of streets, horizontal (or West-East oriented) streets and vertical (or North-South oriented) streets, similar to a Manhattan grid. The horizontal (resp vertical streets) streets have abscissas (resp. ordinates) which are integer multiple of inverse power of two. The number of binary digits after the coma minus indicates the level of the street, starting with the street with abscissas (resp ordinate) $1 / 2$ being at level 0 .

We assume that the streets of level 0 are the highways of the city which support the most traffic, the level 1 are the main streets, the other levels are secondary streets, the traffic decreasing with level number.

The street grid can, of course, be based on on the powers of a different number, for example 3 or 4 . This particular model is realistic for a modern US city. Figure 1 shows a map of Indianapolis as an example. It could also model the pattern of older cities in the ancient world. In this case, the model would display a similar hierarchical street distribution but plugged into a more chaotic geometric pattern instead that of the grid pattern.

\section{B. Hyperfractal Mobile nodes distribution}

It is assumed that the density of mobiles on streets decays as a decreasing function of the street. The process of assigning points to the streets is performed recursively, in iterations, similar to the process for obtaining the Cantor Dust [8].

We notice that the two streets of level 0 form a central cross which splits the map in exactly 4 quadrants. Let us assume a probability $p^{\prime}$ and denote by $q^{\prime}$ the complementary probability $1-p^{\prime}$. A mobile node is dispatched on a street according to the following procedure:

- with probability $p^{\prime}$ the mobile node is located on the cross according to a uniform distribution.

- otherwise, with probability $q^{\prime} / 4$, it is located in one the four quadrants where the assignation procedure continues recursively.

The procedure stops when the mobile node is assigned to a cross of a level $m \geq 0$. A cross of level $m$ consists of two intersecting segments of streets of level $\mathrm{m}$. An example of a decreasing density in the street assignment process performed in $L=4$ steps is given in Figure 2 .

Taking the unit density for the initial map, the density of mobile nodes in a quadrant is $q^{\prime} / 4$. Let $\mu_{H}$ be the density of mobile nodes assigned on a street of level $H$. It satisfies:

$$
\mu_{H}=\left(p^{\prime} / 2\right)\left(q^{\prime} / 2\right)^{H}
$$

The measure (understood in the Lebesgue meaning) which represents the actual density of mobile nodes in the map has strong scaling properties. The most important one is that the map as a whole is identically reproduced in each of the four quadrants but with a weight $q^{\prime} / 4$ instead of 1 . Thus the measure has a structure which recalls the structure of a fractal set, such as the Cantor map. A crucial difference lies

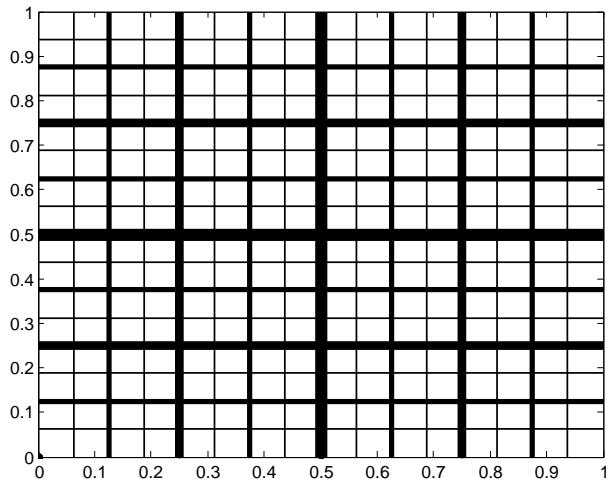

Figure 2: Example of mobile density Map obtained in $L=4$ iterations. The lines are thicker for the level obtained first and become thinner with the increase of the level.

in the fact that its dimension, $d_{m}$, is in fact greater than 2 , the Euclidean dimension. Indeed, considering the map in only half of its length consists into considering the same map but with a reduced weight by a factor $q^{\prime} / 4$. Thus, one obtains:

$$
\left(\frac{1}{2}\right)^{d_{m}}=q^{\prime} / 4
$$

Thus

$$
d_{m}=\frac{\log \left(\frac{4}{q^{\prime}}\right)}{\log 2}>2
$$

This property can only be explained via the concept of measure. We coin here the term hyperfractal to refer to this new kind of self scaling object in euclidean space. Notice that when $p^{\prime} \rightarrow 0$ then $d_{m} \rightarrow 2$ and the measure tends to the uniform measure in the unit square.

One could extend the map in the whole quarter of the plane. In this case the map is made of half infinite horizontal and vertical streets. If a vertical (resp. horizontal) street has level $H$, it contains mobile nodes with uniform density $\mu_{H}=\left(p^{\prime} / 2\right)\left(q^{\prime} / 2\right)^{H}$.

\section{Canyon effect and relays}

Due to the presence of buildings, the radio wave can hardly propagate beyond the streets borders. The buildings are made of concrete, glass and steal which generate a formidable obstacle for propagation. Therefore, we adopt the canyon propagation model where the signal emitted by a mobile node propagates only on the axis where it stands on. Considering the given construction process, the probability that a mobile node is placed in an intersection tends to zero and mobiles positioned on two different streets will never be able to communicate. Therefore, one needs to add relays in some street crossings in oder to guarantee connectivity and packet delivery.

A relay consists of two connected wireless devices: a first one transmitting and receiving on the North-South axis, and the second one, transmitting and receiving on West-East axis. We again make use of a hyperfractal process to select the intersections which will contain a relay. 


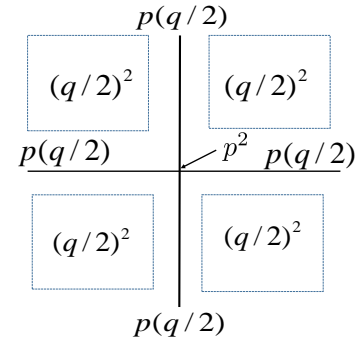

Figure 3: Relays placement process

Denote by $p$ a fixed probability and $q=1-p$ the complementary probability. A run for selecting a street crossing requires two processes: the in-quadrant process and the insegment process. The selection starts with the in-quadrant process as follows:

- with probability $p^{2}$, the selection is the central crossing of the two streets of level 0 ;

- with probability $p(q / 2)$, the relay is placed in one of the four street segments of level 0 starting at this point: North, South, West or East, and the process continues on the segment with the in-segment process

- with probability $(q / 2)^{2}$, the relay is placed in one of the four quadrants delimited by the central cross and the inquadrant process continues recursively.

The process of placing the relays is illustrated in Fig. 3. We perform $M$ independent runs of selection. If one crossing is selected several times (e.g. the central crossing), only one relay will be installed in the respective crossing. This reduction will mean that the number of actually placed relays will be much smaller than $M$.

Following a reasoning similar to the mobile placement, the relay placement is hyperfractal with a hyperfractal dimension $d_{r}$ :

$$
d_{r}=2 \frac{\log (2 / q)}{\log 2} .
$$

Let $p(H, V)$ be the probability that the run selects a crossing of two streets, one horizontal street of level $H$ and one vertical street of level $V$. There are $2^{H+V}$ of such crossings. We have:

$$
p(H, V)=p^{2}(q / 2)^{H+V} .
$$

Thus the probability that such crossing is selected to host a relay is $1-(1-p(H, V))^{M}$. When $M$ is large, the probability is approximately $1-\exp (-M p(H, V))$. If the number of crossing selection run is a Poisson random variable of mean $\rho$, then the probability that a crossing hosts a relay is exactly $1-\exp (-M p(H, V))$. The relay Poisson model is interesting as it generates independent crossings. For this reason, we keep the relay Poisson model from now on.

The average number of relays on a streets of level $H$ is denoted by $L_{H}(\rho)$ and satisfies the identity:

$$
L_{H}(\rho)=\sum_{V \geq 0} 2^{V}(1-\exp (-p(H, V) \rho)) .
$$

We notice that $L_{H}(\rho)=L_{0}\left((q / 2)^{H} \rho\right)$ and that $L_{0}(\rho)$ satisfies the functional equation:

$$
L_{0}(\rho)=1-\exp \left(-p^{2} \rho\right)+2 L_{0}((q / 2) \rho) .
$$

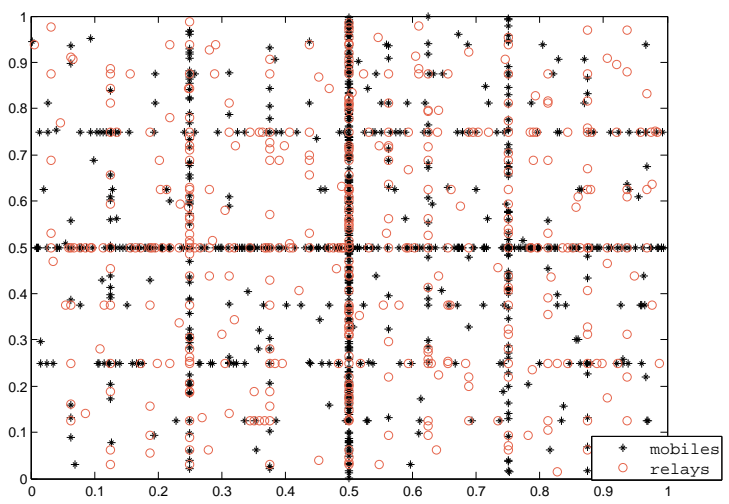

Figure 4: Hyperfractal map of level 10 with mobiles and relays, $d_{m}=3, d_{r}=3$

It is known from [9], [10] that this classic equation has a solution such as $L_{0}(\rho)=O\left(\rho^{2 / d_{r}}\right)$.

The average total number of relays in the city, $R(\rho)$, has the expression:

$$
R(\rho)=\sum_{H, V \geq 0} 2^{H+V}(1-\exp (-p(H, V) \rho))
$$

and satisfies the functional equation

$$
R(\rho)=1-\exp \left(-p^{2} \rho\right)+4 L_{0}(p(q / 2) \rho)+4 R\left((q / 2)^{2} \rho\right) .
$$

From the same reference, [9], [10], one gets

$$
R(\rho)=O\left(\rho^{2 / d_{r}} \log \rho\right)
$$

Since $2 / d_{r}<1$ the number of relays is much smaller than $\rho$. In the following we will take $\rho=O(N)$. Due to lack of space, complete proofs will be given in a extended version of the paper.

Given the process of construction, the probability of existence of mobile nodes is independent of the probability of existence of relays. Furthermore, the propagation limited to the axes does not ensure full connectivity. The connectivity graph presents a giant component. A complete Hyperfractal map containing both mobile nodes and relays is presented in Fig. 4.

\section{ROUTING AND CAPACITY}

Here it is considered that the nodes in the network, both mobile nodes and relays, communicate like in a mobile ad hoc network where packets are routed forward from their sources to their destinations. We consider a table driven routing where each nodes looks into a routing table to determine the next relay to send the packet. The distributed protocols needed to construct the routing tables in every node is not taken into account. To simplify, we consider that the routing tables are built by a central entity which has a full knowledge of the topology of the network as well as the precise locations of the nodes.

The routing table will be computed according to a minimum cost path over a cost matrix $\left[t_{i j}\right]$ where $t_{i j}$ represents the cost of directly transmitting a packet from node $i$ to node $j$. The min cost path from node $i$ to node $j$ which optimizes the 
relaying nodes (either mobile nodes or fixed relays) is denoted $m_{i j}$ and satisfies:

$$
m_{i j}=\min _{k}\left\{m_{i k}+t_{k j}\right\}, \forall(i, j),
$$

Due to the canyon effect some nodes can be disconnected from the rest of the network, several connected components may appear and some routes may not exist. In the case node $i$ and node $j$ cannot communicate $m_{i j}=\infty$. We restrict our analysis to the giant component of the network which contains the central node $\left[\frac{1}{2}, \frac{1}{2}\right]$. We know that the size of this giant component is strictly of order $N$.

In this paper two routing strategies are considered:

- the nearest neighbor routing;

- the minimum delay routing.

To simplify, it is assumed that the channel is noiseless, i.e. every node is reachable given it is aligned (i.e. on direction North-South or West-East) regardless of the distance towards the transmitter. However, due to interference from other transmitters, the quality of the connection may significantly drop with respect to the relative distance between the nodes.

1) The nearest neighbor routing: In this strategy the next relay is always a next neighbor on an axis, i.e. there exist no other nodes between the transmitter and the receiver. Thus

$$
\begin{cases}t_{i j}=1 & \text { if nodes } i \text { and } j \text { are aligned } \\
& \text { and } \nexists k \text { such that } d(i, j)=d(i, k)+d(k, j) \\
t_{i j}=\infty & \text { otherwise }\end{cases}
$$

This formula implies that, although the farther nodes on the axis might be reachable, the interference created by the nearest neighbors gives the farther node an infinite cost.

2) The minimum delay routing: In this model, the underlying medium access control is slotted Aloha with per slot and per node transmission probability $p_{A}$. Considering interference, required SIR, and attenuation factors, we denote $p_{i j}$ the probability that node $j$ correctly receives a packet transmission from node $i$ at a given slot. Clearly, $p_{i j} \leq$ $p_{A}\left(1-p_{A}\right)$, since a required condition is that node $i$ transmits and node $j$ does not. Therefore, the average delay required for node $i$ to successfully transmit a packet to node $j$ is $t_{i j}=1 / p_{i j}$. The quantity $m_{i j}$ will be the cost of the minimum average path delay .

One should notice that the alternative strategy where we consider the minimum average number of retransmission on the path to the destination will provide the same shortest path. Indeed it will consist into multiplying the coefficients $t_{i j}$ and $m_{i j}$ by the factor $p_{A}$.

\section{A. Capacity}

In this section a known result [11] for the throughput capacity of the ad-hoc networks is reminded and extended. Let us remind the following notations and results. For the hyperfractal distribution of nodes we assume $N$ mobile nodes and that the relay nodes distribution satisfies $\rho=N$. It is already known that $R(N)=o(N)$. Denote by $\mathcal{G}_{N}$ the giant component of the network, its size $\left|\mathcal{G}_{N}\right|$ is $\Theta(N)$.
Let $\zeta(N)$ be the throughput capacity, defined as the expected number of packets delivered to their destinations per slot. It is a metric that depends on the number of nodes, $N$, SIR threshold, $K$, attenuation coefficient. $\alpha$, the medium access scheme parameters and the expected transmission rate of each node, $\Omega_{i}(N)$.

Theorem 1. The throughput capacity of random wireless networks is of order [11]:

$$
\zeta(N)=\Theta\left(\frac{N^{2} \sum_{i \in \mathcal{G}_{N}} \Omega_{i}(N)}{\sum_{i, j \in \mathcal{G}_{N}} m_{i j}}\right) .
$$

We intentionally keep vague the question whether or not the relay nodes should be mentioned or not in the giant component or if those relays generate traffic or limit their action to forwarding packets generated by mobile nodes. In any case this does not change the order of magnitude of the global throughput estimate.

One can notice that the quantity:

$$
D_{N}=\frac{\sum_{i, j \in \mathcal{G}_{N}} m_{i j}}{\left|\mathcal{G}_{N}\right|\left(\mid \mathcal{G}_{N}-1\right)}
$$

is the average path cost in the giant component.

In the nearest neighbor routing it is assumed that all nodes require the same quantity of bandwidth $\beta: \forall i: \Omega_{i}=\beta$. With Aloha and with the min path cost we have exactly $\forall i: \Omega_{i}=$ $p_{A}$ slot bandwidth per node. To simplify we, it is assumed that the nodes transmit something even if they have no packet in their buffer. A complete proof of this results can be found in [11].

\section{B. Average path cost and capacity estimate}

In the context of nearest neighbor routing strategy, we prove the following result:

Theorem 2. The average number of hops in a Hyperfractal is:

$$
D_{N}=O\left(N^{1-\frac{2}{\left(1+1 / d_{m}\right) d_{r}}}\right)
$$

where $N$ is the number of mobile nodes and $d_{m}$ and $d_{r}$ are respectively the hyperfractal dimensions of mobile nodes and relays.

We conjecture (supported by the simulations) that with the minimum delay routing strategy using Aloha we have the same scaling.

Conjecture 2.1. The average path cost in minimum delay routing in a Hyperfractal scales as:

$$
D_{N}=O\left(N^{1-\frac{2}{\left(1+1 / d_{m}\right) d_{r}}}\right)
$$

Notice that when $d_{r} \rightarrow 2$ (i.e. when the relay distribution tends to be uniform Poisson) then $D_{N}$ tends to be in $N^{\frac{1}{1+d_{m}}}$ which is $o\left(N^{1 / 3}\right)$ much smaller than the average cost with uniform Poisson shot model, where the average path cost is $O\left(N^{1 / 2}\right)$ in the plane [4]. In fact $N^{1 / 3}$ would be the order of magnitude of the average path length in uniform Poisson in a cube. The estimate $D_{N}=o\left(N^{1 /\left(1+d_{m}\right)}\right)$ suggests the idea 
that the hyperfractal distribution operates as if the distribution of mobile nodes was a uniform distribution in an hypercube of dimension $1+d_{m}$.

Corollary 2.1. The capacity in a Hyperfractal with $N$ mobile nodes scales as:

$$
\zeta(N)=\Theta\left(N^{\frac{2}{(1+1 / d m) d r}}\right)
$$

In consequence, when $d_{r} \rightarrow 2$ and $d_{m} \rightarrow \infty$ the capacity tends to scale linearly.

\section{PROOFS OF THEOREM 2}

Proof. The proof will be left sketchy due to the lack of room. Mobile node $m H$ situated on a line of level $H$ wants to send a packet to its destination, $m V$, situated on a line of level $V$. The largest order of magnitude for path cost occurs when $m V$ and $m_{H}$ are on non parallel streets as illustrated in Figure 5,a). Furthermore, the dominant case is $V=H=0$, the other cases will just introduce extra factors which will not change the obtained orders of magnitude.

Given that the densities of the population on the support street of mobile nodes are high, the game will consist into diverting the packet by following a vertical line of level $x>0$ with a much lower density. A similar phenomenon happens towards mobile $m V$, the packet will return on the support street of $m V$ as "close" as possible to node $m V$, after following an horizontal street of level $y>0$. Since it is considered $V=H$, by symmetry we will only consider that $x=y$.

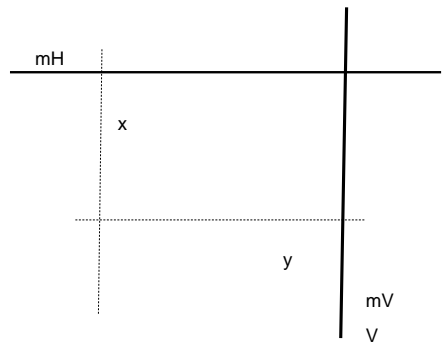

a)

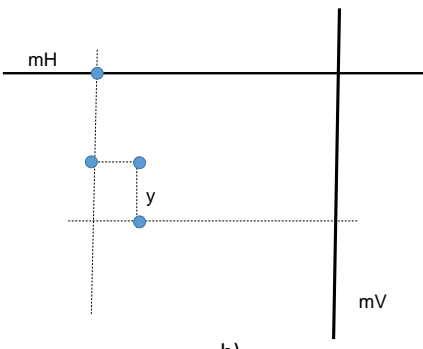

b)
Figure 5: Routing in a Hyperfractal a) intermediate levels $x$ and $\mathrm{y}, \mathrm{b}$ )extra intermediate levels

In order for the packet to change direction in its route, it is mandatory that a relay exists at the crossing. Let $L(x, y)$ be the average distance between a random mobile node on a street of level $y$ to the first relay to a street of level $x$. Every crossing between streets of level $x$ and $y$ is independent and holds a relay with probability $1-\exp (-\rho p(x, y))$. Since such crossings are regularly spaced by interval $2^{-x}$ we get:

$$
L(x, y) \leq \frac{2^{-x}}{1-\exp (-\rho p(x, y))}
$$

Our aim is now to count the number of nodes traversed by the packet on its route. There is no room to prove that the main contribution in the count comes from the number of traversed mobile nodes. It is first assumed that the two streets of level $x$ have a relay at their intersection. In this case, the average number of traversed nodes is upper bounded by $2 N \mu_{0} L(x, 0)+2 N \mu_{x}$.

$$
\begin{aligned}
& \text { If } x=\alpha \frac{\log \rho}{\log (2 / q)} \text { for } 1 / 2<\alpha<1 \text {, then } \\
& \qquad L(x, 0)=\frac{\rho^{-2 \alpha / d_{r}}}{1-\exp \left(-p^{2} \rho^{1-\alpha}\right)}=O\left(\rho^{-2 \alpha / d_{r}}\right) .
\end{aligned}
$$

We also have $\mu_{x}=O\left(\rho^{-\alpha \frac{\log \left(2 / q^{\prime}\right)}{\log (2 / q)}}\right)=o\left(\rho^{-2 \alpha / d_{r}}\right)$. The probability that there exists a valid relay at level $x$ street intersection is $1-\exp (-\rho p(x, x)))$ which tends to zero when $\alpha>1 / 2$ since $\rho p(x, x)=p^{2} \rho^{1-2 \alpha}$. Let us assume this holds (to be verified afterward).

Following this observation, an intermediate level, $0<y<$ $x$, is added to the route with:

$$
y=\beta \frac{\log \rho}{\log (2 / q)}
$$

with $\beta=1-\alpha$. We consider the first relays on the streets of level $y$ counted from the intersection of the streets, they are at average distance $L(y, x)$. The probability that the intersection two streets of level $y$ has a relay is $1-\exp (-\rho p(y, y))$ which tends to 1 when $\rho \rightarrow \infty$ since $\rho p(y, y)=p^{2} \rho^{1-2 \beta}$.

The average distance between the intersection $(x, x)$ with the routes of levels $y$ which have a valid relay at their intersection is $\frac{L(y, x)}{1-\exp (-\rho p(y, y))}=O\left(\rho^{-2 \beta / d_{r}}\right)$.

Level $y$ adds a number of hops of an average quantity $2 N \mu_{y} \frac{L(x, y)}{1-\exp (-\rho p(y, y))}$ thus the number of extra hops if of order: $O\left(N \rho^{-2 \beta \frac{d_{m}}{d_{r}}}\right)$. The total number of hops is now $O\left(N \rho^{-2 \alpha / d_{r}}\right)+O\left(N \rho^{-2 \beta \frac{d_{m}}{d_{r}}}\right)$. This quantity is minimized for $\alpha=\frac{d_{m}}{1+d_{m}}$ and $\beta=\frac{1}{1+d_{m}}$. We confirm that $\alpha>1 / 2$

The minimized value of the number of hops is, thus:

$$
D_{N}=O\left(N \rho^{-\frac{2}{\left(1+1 / d_{m}\right) d_{r}}}\right)
$$

\section{NumericAl RESUltS}

\section{A. Aloha model with Rayleigh fading}

We consider slotted, synchronized Aloha scheme and that all nodes are backlogged. Each transmitting node uses the same nominal transmit power. Path-loss between node $i$ and node $j$ is modeled by the power-law function $l(i, j)=(A r)^{\alpha}$, when the two nodes $i$ and $j$ are aligned, where $A, \alpha$ are some constants and $r$ is the distance between transmitter and receiver, otherwise $l(i, j)=0$. The reception undergoes Rayleigh fading $F_{i j}$ independent over nodes and time, the signal received by receiver $j$ from transmitter $i$ at time slot $n$ becomes $F_{i j} l(i, j)$.

By assuming the background noise power negligible, and that node $i$ is in transmit mode, the successful reception of a signal transmitted from node $i$ to a node $j$ at a given time slot occurs when:

$$
F_{i j} l(i, j)>K \sum_{k \in B-\{i\}} F_{k j} l(k, j)
$$



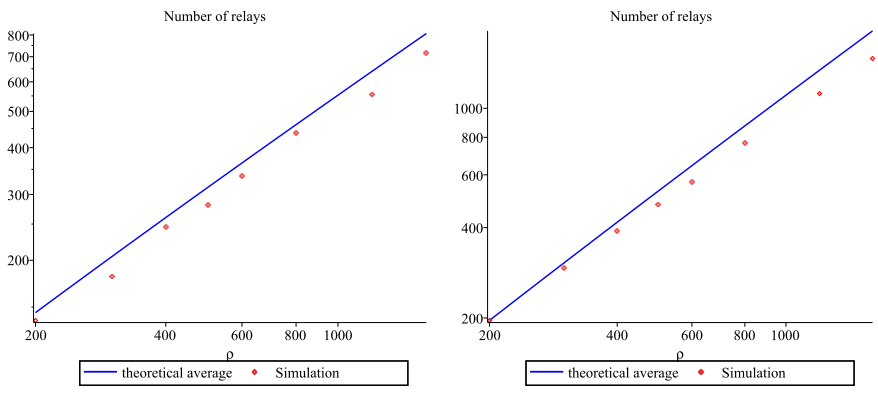

Figure 6: Average number of equipped intersections for the two configurations, a) $d_{m}=d_{r}=3$, b) $d_{m}=3, d_{r}=2.2$

where $K$ is the SIR threshold related to the bit-rate when a particular modulation plus coding scheme is considered. $B$ is the subset of nodes transmitting at the considered time slot. In the particular case of a relay we have to separate the signals coming from the vertical street with the signals received from the horizontal streets

In order to avoid computational expensive and timeconsuming simulations of ALOHA protocol, we use the following results for computing the probabilities of successful reception, $p_{i j}$ when independent Rayleigh fading is applied:

$$
p_{i j}=p_{A}\left(1-p_{A}\right) \prod_{k \neq i, j} w_{k j}(K / l(i, j)) .
$$

where $w_{k j}(\theta)$ is the Laplace transform of the signal produced by node $k$ over node $j$, these quantities are simple rational functions of $\theta$. We will take $\alpha=4$ and $K=1$.

Now that we have this result on the probability of successful reception, we apply Dijkstra algorithm on the cost matrix $t_{i j}=$ $1 / p_{i j}$. Two configurations are studied, one where the fractal dimension of mobiles is equal to the ones of relays, $d_{m}=d_{r}$ and the second one, where the two values differ, $d_{m}>d_{r}$.

\section{B. Simulation results}

Figure 6 validates the scaling law for the average number of equipped intersection introduced in equation (10) for both evaluation setups. Figure 7 validates the Conjecture 2.1 by simulations and computation using minimum path cost algorithm. In fact it shows that the proposed scaling law is rather pessimistic, offering improvement for future work.

Another simulation is performed on the throughout capacity. Thanks to the given closed form expressions, computations can be done fast and conveniently. We compute the throughput capacity using Theorem 1 where the quantities $m_{i, j}$ are obtained by using a minimum path cost computation algorithm, where the values in the cost matrix are the inverse of the expression [22].

Figure 8 illustrates the results of this computation. Again, the conjectured scaling law is pessimistic.

\section{CONCLUSION}

This work introduced a new model, which called "Hyperfractal", for the ad hoc urban wireless networks. The model

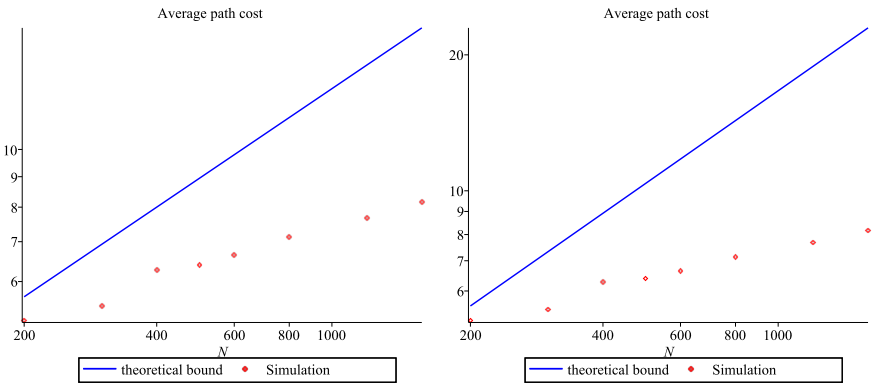

Figure 7: Average path cost scaling for the two configurations, a) $d_{m}=d_{r}=3$, b) $d_{m}=3, d_{r}=2.2$
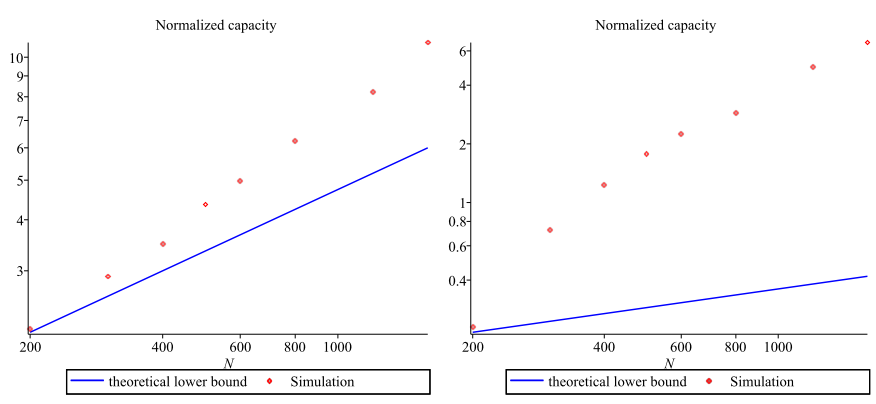

Figure 8: Throughput capacity scaling for the two configurations, a) $d_{m}=d_{r}=3$, b) $d_{m}=3, d_{r}=2.2$

captures the irregularity and variability of the node configuration and, in addition to previous works, the self-similarity of the topology. The hyperfractal model is a Poisson shot model which has support a measure with scaling properties. We showed here the scaling of metrics of interest like throughput capacity and the number of hops and path cost under two routing algorithms. The results show that the scaling is much better than with the classic Poisson shot model.

\section{REFERENCES}

[1] B. Blaszczyszyn and P. Muhlethaler, "Random linear multihop relaying in a general field of interferers using spatial aloha," IEEE Transactions on Wireless Communications, July 2015.

[2] D. Herculea, C. S. Chen, M. Haddad, and V. Capdevielle, "Straight: Stochastic geometry and user history based mobility estimation," in HotPOST, 2016.

[3] F. Baccelli and B. Blaszczyszyn, Stochastic Geometry and Wireless Networks, Volume I - Theory. NoW Publishers.

[4] P. Gupta and P. R. Kumar, "The capacity of wireless networks," IEEE Transactions Information Theory, pp. 388-404, 2006.

[5] M. Karabacak and all., "Mobility performance of macrocell-assisted small cells in manhattan model," in VTC, May 2014.

[6] P. Jacquet, "Optimized outage capacity in random wireless networks in uniform and fractal maps," in ISIT, June 2015, pp. 166-170.

[7] — - "Capacity of simple multiple-input-single-output wireless networks over uniform or fractal maps," in MASCOTS, Aug 2013.

[8] Wikipedia, "Cantor set - wikipedia, the free encyclopedia," 2016.

[9] P. Flajolet, X. Gourdon, and P. Dumas, "Mellin transforms and asymptotics: Harmonic sums," Theoretical Computer Science, 1995.

[10] P. Jacquet and W. Szpankowski, "Analytical depoissonization and its applications," Theoretical Computer Science.

[11] S. Malik, P. Jacquet, and C. Adjih, "On the throughput capacity of wireless multi-hop networks with aloha, node coloring and csma," in Wireless Days, Oct 2011. 